\title{
Loudspeaker Equalization with Post-Processing
}

\author{
Wee Ser \\ Center for Signal Processing, School of Electrical \& Electronic Engineering, Nanyang Technological University, \\ Singapore 639798, Singapore \\ Email:ewser@ntu.edu.sg \\ Peng Wang \\ Center for Signal Processing, School of Electrical \& Electronic Engineering, Nanyang Technological University, \\ Singapore 639798, Singapore \\ Email: ewangp@ntu.edu.sg

\section{Ming Zhang} \\ Center for Signal Processing, School of Electrical \& Electronic Engineering, Nanyang Technological University, \\ Singapore 639798, Singapore \\ Email:mzhang21@hotmail.com
}

Received 1 November 2001 and in revised form 23 June 2002

Loudspeaker equalization is an essential technique in audio system design. A well-known equalization scheme is based on the deconvolution of the desired equalized response with the measured impulse response of the loudspeaker. In this paper, a postprocessing scheme is combined with the deconvolution-based algorithm to provide a better equalization effect. Computer simulation results are given to demonstrate the significant improvement that can be achieved using this method.

Keywords and phrases: loudspeaker equalizer, post-processing.

\section{INTRODUCTION}

A loudspeaker is a device that converts a signal from its electric form to audible sound wave. Noticeable distortions may be introduced in this conversion leading to a significant loss of sound quality. The technique of loudspeaker equalization has thus been developed to deal with this problem. Among all the equalization schemes presented in the literature $[1,2,3,4,5,6,7,8,9,10,11,12,13]$, the deconvolutionbased scheme [1] has the simplest filter structure and is widely used. However, this scheme does not work effectively at low and middle frequencies. Multiband approach overcomes this shortcoming and is able to provide satisfactory equalization for the entire frequency band of interest $[2,3]$, but its complex structure prevents it from being used in low-cost systems. In our previous work reported in $[4,5]$, a warped filter has been incorporated into the multiband equalizer design to reduce the structural complexity. In this paper, a different design approach is investigated. By suitably combining the deconvolution-based equalizer with a postprocessing technique, a better equalization effect can be obtained with a marginal increase in the structural complexity.

This paper is organized as follows. In Section 2, the deconvolution-based equalization scheme is briefly described and the principle of the proposed equalization scheme with post-processing is introduced. In Section 3, the optimization process for the proposed scheme is discussed. Computer simulations have been conducted to demonstrate the effectiveness of the proposed scheme, and the results are given in Section 4. The paper ends with the concluding remarks of Section 5 .

\section{PRINCIPLE OF THE PROPOSED SCHEME}

Loudspeaker equalization involves the inverse filtering of the signal that is distorted by the loudspeaker. In the simplest case, it can be depicted as a convolution process, that is,

$$
g(n) * h(n)=z(n-\tau)+e(n),
$$

where $h(n)$ and $g(n)$ are the impulse responses of the loudspeaker and the equalizer, respectively, $z(n)$ is the desired equalized response, $e(n)$ is the residue error, and $\tau$ is the time delay incurred due to the mixed-phase nature of the loudspeaker $[1,6,14]$.

Mourjopoulos introduced the least-mean-square algorithm into loudspeaker equalizer design and developed the deconvolution-based scheme [1]. The equalizer filter obtained from this scheme is optimum in the sense that it is 
able to minimize the mean square error

$$
\begin{aligned}
E_{s} & =\sum_{n=0}^{N+M-2} e^{2}(n) \\
& =\sum_{n=0}^{N+M-2}\left[z(n-\tau)-\sum_{m=\max (0, n-M+1)}^{\min (n, N-1)} g(m) h(n-m)\right]^{2},
\end{aligned}
$$

where $N$ is the length of $g(n)$ and $M$ is the length of $h(n)$. Taking $(\partial / \partial g(i)) E_{s}=0,(i=0,1, \ldots, N-1)$, the matrix form of the system equation can be derived

$$
\mathbf{g}=\mathbf{H}^{\#} \mathbf{z}
$$

where

$$
\mathbf{g}=\left[\begin{array}{lllll}
g(0) & g(1) & g(2) & \cdots & g(N-1)
\end{array}\right]^{T}
$$

is the coefficient vector of the equalizer filter,

$\mathbf{z}=\left[\begin{array}{lllllllllll}0 & \cdots & 0 & z(0) & z(1) & z(2) & \cdots & z(P-1) & 0 & \cdots & 0\end{array}\right]^{T}$

is an $(N+M-1) \times 1$ vector, $P$ is the length of the desired impulse response $z(n)$, \# denotes pseudo inverse operation and

$$
\mathbf{H}^{\#}=\left(\mathbf{H}^{T} \mathbf{H}\right)^{-1} \mathbf{H}^{T}
$$

and $\mathbf{H}$ is an $(N+M-1) \times N$ matrix defined by

$$
\mathbf{H}=\left[\begin{array}{cccc}
h_{0} & 0 & \cdots & 0 \\
h_{1} & h_{0} & \cdots & 0 \\
\vdots & \vdots & \ddots & \vdots \\
h_{N-1} & h_{N-2} & \cdots & h_{0} \\
\vdots & \vdots & \ddots & \vdots \\
h_{M-1} & h_{M-2} & \cdots & h_{M-N} \\
0 & h_{M-1} & \cdots & h_{M-N+1} \\
\vdots & \vdots & \ddots & \vdots \\
0 & 0 & \cdots & h_{M-1}
\end{array}\right]
$$

As can be seen from Figure $2 b$, noticeable distortions can still be observed in the equalized frequency response using this deconvolution-based scheme, especially at low and middle frequencies. In this study, a new equalization method incorporating a post-processing technique is proposed to partially solve this problem. The principle of this method is to modify the desired equalized response according to the residue error observed from the previous equalization process.

Supposing $z^{\prime}(n)$ is the modified desired-equalizedresponse and $g^{\prime}(n)$ is the corresponding new equalizer design, $g^{\prime}(n)$ can be derived by replacing $\mathbf{z}$ with $\mathbf{z}^{\prime}$ in (3). The equalization process can be written accordingly as

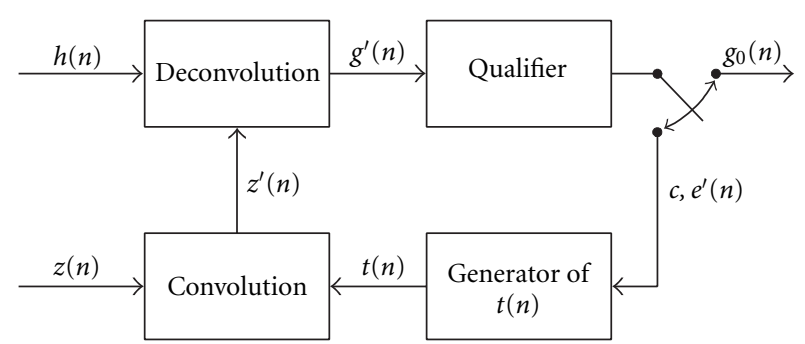

FIGURE 1: Flow chart of the iterative process.

follows

$$
g^{\prime}(n) * h(n)=z^{\prime}(n-\tau)+e^{\prime}(n),
$$

where $e^{\prime}(n)$ is the new residue error. The modified desiredequalized-response, $z^{\prime}(n)$, is obtained by filtering $z(n)$ with an adjustment function $t(n)$, that is,

$$
z^{\prime}(n)=z(n) * t(n)
$$

In order to obtain maximum suppression of the residue error, the adjustment function, $t(n)$, must be designed very carefully. In this study, it is determined as follows:

Let $Y(l)$ be the Fourier transformation of the equalized impulse response, that is,

$$
Y(l)=\mathscr{F}[g(n) * h(n)]
$$

where $\mathscr{F}$ denotes Fourier transform. The smoothened function of $Y(l)$ is defined as

$$
\tilde{Y}(l)=\sum_{i=-K}^{K} \frac{Y(l+i)}{2 K+1},
$$

where $K$ is a positive integer. The adjustment function can now be obtained as follows,

$$
t(n)=\mathscr{F}^{-1}[a /|\tilde{Y}(l)|]^{c},
$$

where $\mathscr{F}^{-1}$ denotes the inverse Fourier transform, $a$ is the mean value of $\tilde{Y}(l)$, and $c$ is a positive adjustment parameter. It can be observed that the adjustment function has been designed such that the strong components of $Y(l)$ are suppressed while its weak components are enhanced.

\section{OPTIMIZATION OF THE PROPOSED SCHEME}

The effect of the proposed equalization method is dominantly determined by the value of the adjustment parameter $c$ in (12). An iterative process has been developed to search for the optimum value for this adjustment parameter.

The flow chart of the iterative process is shown in Figure 1, where $g_{0}(n)$ is the resultant optimum equalizer, and the qualifier is used to decide when to terminate the iteration.

Different qualifications can be devised according to different requirements. In this paper, the normalized variance 


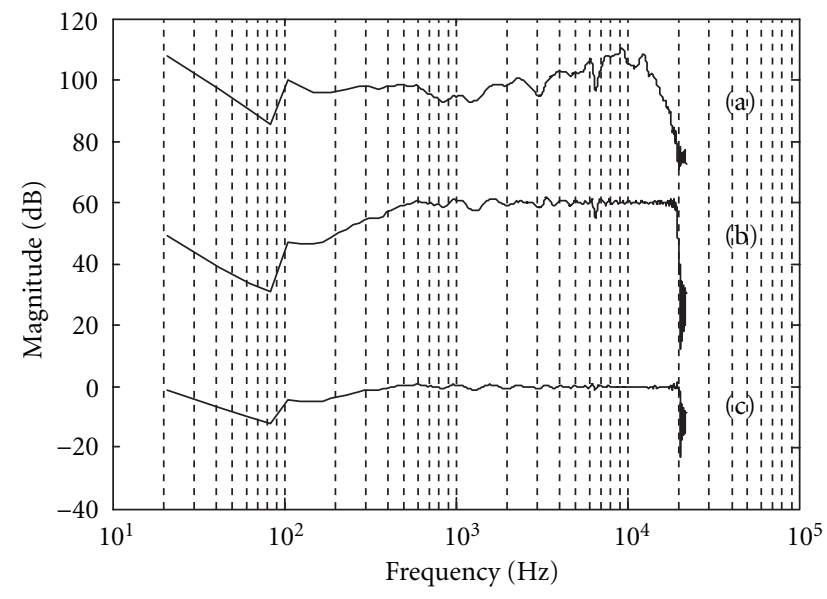

FIgURE 2: Magnitude frequency responses. (a) Measured response of the loudspeaker. (b) Equalized response using the deconvolutionbased scheme. (c) Equalized response using the proposed scheme.

of the magnitude distortion is used to maximize the flatness of the equalized response within the passband, that is,

$$
C_{e}=\frac{1}{L} \sum_{k=0}^{L-1}|[E(k)-\bar{E}] / \bar{E}|^{2}
$$

where $E(k)$ is the magnitude distortion function, that is, $E(k)=\mathscr{F}[e(n)], L$ is the passband width in taps, and $\bar{E}$ is the mean value of $E(k)$ within the passband. A simple optimization scheme using this qualification is summarized as follows:

Step 1. Set the initial value of $c$ to zero, corresponding to the case where there is no adjustment made to the desired equalized response.

Step 2. Design a deconvolution-based equalizer using (3).

Step 3. Calculate $C_{e}$ using (13), which is to be minimized in the subsequent process.

Step 4. Increase the value of $c$ by a certain step size, and calculate $z^{\prime}(n)$ using (9), (10), (11), and (12).

Step 5. Calculate $g^{\prime}(n)$ and the corresponding $C_{e}$.

Step 6. Repeat Steps 4 and 5 until $C_{e}$ is greater than its previous value.

The proposed optimum equalizer has a similar structure to that of the deconvolution-based method, and as such the latter has been used at the initial stage of the iteration to accelerate the iterative process.

\section{COMPUTER SIMULATIONS}

Computer simulations have been conducted to test the effectiveness of the proposed scheme. The results of a simula- tion carried out on a low-cost desktop loudspeaker are shown in Figure 2, where (a) is the measured frequency response of the loudspeaker, (b) is the equalized frequency response using the deconvolution-based scheme, and (c) is that obtained using the proposed scheme. The desired equalized response, $z(n)$, has been modeled as one having a linear phase and a flat magnitude property within the passband $(0 \sim 20 \mathrm{kHz})$. The optimization method described in Section 3 has been employed in the equalizer design to minimize $C_{e}$. In this simulation, the length of all the equalizer filters has been set to 100 . It should be noted that the purpose of the proposed scheme is to redistribute the limited resources that can be used to construct the equalizer so as to achieve a better equalization effect. If the filter length is sufficiently large (e.g., 1000 for the loudspeaker used in this simulation), the deconvolutionbased method alone is able to provide a good equalization effect on the entire frequency band, and the improvement contributed by the post-processing scheme may not be obvious.

As can be observed from the figure, the magnitude frequency response of the loudspeaker contains noticeable distortion. The deconvolution-based equalization helps to reduce the distortion greatly, but the resultant equalized response is still poor at low and middle frequencies. Using the proposed scheme, an overall better equalization effect is obtained. In Table 1, the values of $C_{e}$ (equation (13)) for the two equalized responses are compared to further illustrate the improvement.

TABLE 1: Comparison of normalized variance of the magnitude distortion.

\begin{tabular}{lcc}
\hline & $\begin{array}{c}\text { Deconvolution-based } \\
\text { equalization }\end{array}$ & $\begin{array}{c}\text { Equalization with } \\
\text { post-processing }\end{array}$ \\
\hline$C_{e}(\mathrm{~dB})$ & -14.9394 & -19.1804 \\
\hline
\end{tabular}

TABLE 2: Comparison of equalization effects using different qualifications.

\begin{tabular}{ccc}
\hline & Using $C_{e}$ as qualification & Using $C_{e L}$ as qualification \\
\hline$C_{e}(\mathrm{~dB})$ & -19.1804 & -18.4319 \\
$C_{e L}(\mathrm{~dB})$ & -18.3299 & -19.7102 \\
\hline
\end{tabular}

Qualifications other than $C_{e}$ can be adopted in the optimization process to realize different equalization effects. An example is given in Table 2, where the normalized variance of the magnitude distortion at low frequencies, $C_{e L}$, is used to further improve the equalization effect at low frequencies. $C_{e L}$ can be expressed as

$$
C_{e L}=\frac{1}{I} \sum_{k=0}^{I-1}|[E(k)-\bar{E}] / \bar{E}|^{2},
$$

where $I$ is the upper boundary of the low frequency band. As is illustrated, the distortion at low frequencies $(0 \sim 4 \mathrm{kHz}$ 
in this paper) is further suppressed, but the overall equalization effect is deteriorated compared with that using $C_{e}$ as the qualifier in the optimization.

It should be noted that in the definition of the adjustment function given in (12), all frequency components of the equalized response are enhanced or suppressed at the same exponential rate of $c$. A reasonable conjecture is that, by allowing these frequency components to vary more freely, the resultant equalization effect could be improved further. This conjecture has been substantiated with a simple test, where the entire frequency band is divided into two sub-bands and the steepest decent algorithm is applied to search for the optimum two-element adjustment parameter vector that minimizes $C_{e}$. For the loudspeaker used in Figure 2, the magnitude fluctuation of the equalized response within the passband is reduced further by about $0.8 \mathrm{~dB}$, compared with that using a uniform adjustment parameter (from $-19.1804 \mathrm{~dB}$ to $-20.0347 \mathrm{~dB}$ ).

\section{CONCLUSIONS AND REMARKS}

In this paper, a new loudspeaker equalization method is proposed. By using the proposed post-processing technique to refine the result of the classical deconvolution-based design, the magnitude fluctuation of the equalized response within the passband can be further reduced. The effectiveness has been illustrated through computer simulations. Unlike other methods such as the multiband or warped filter equalization, the proposed scheme has a relatively simple implementation design, which makes it very attractive for low-cost loudspeaker design. This method can also be incorporated into the multiband or warped filter solutions to provide better equalization effect.

\section{REFERENCES}

[1] J. Mourjopoulos, "Digital equalization methods for audio systems," presented at the 84th Convention of the Audio Engineering Society, Paris, France, preprint 2598, Journal of the Audio Engineering Society (abstract), vol. 36, pp. 384, May, 1988.

[2] J. A. Henriquez, T. E. Riemer, and R. E. Trahan Jr., "A phaselinear audio equalizer: design and implementation," Journal of the Audio Engineering Society, vol. 38, no. 9, pp. 653-666, 1990.

[3] J. A. Jensen, "A new principle for an all-digital preamplifier and equalizer," Journal of the Audio Engineering Society (Engineering Reports), vol. 35, no. 12, pp. 994-1003, 1987.

[4] P. Wang, W. Ser, and M. Zhang, "A novel dual-band equalizer design for loudspeaker systems," Journal of the Audio Engineering Society, vol. 48, no. 10, pp. 917-921, 2000.

[5] P. Wang, W. Ser, and M. Zhang, "Multiband warped filter equalizer design for loudspeaker systems," in Proc. IEEE Int. Conf. Acoustics, Speech, Signal Processing, pp. 913-916, Istanbul, Turkey, June 2000.

[6] R. Greenfield and M. O. Hawksford, "Efficient filter design for loudspeaker equalization," Journal of the Audio Engineering Society, vol. 39, no. 10, pp. 739-751, November 1991.

[7] W. Ser, M. Zhang, and K. C. Tan, "Practical loudspeaker equalization using digital filter," in Proc. ISSPR '98, Hong Kong, China, September 1998.
[8] G. Karachalios, D. Tsoukalas, and J. Mourjopoulos, "Multiband analysis and equalization of loudspeaker responses," presented at the 98th Convention of the Audio Engineering Society, Paris, France, February, 1995, preprint 3975.

[9] K. Steiglitz, "A note on variable recursive digital filters," IEEE Trans. Acoustics, Speech, and Signal Processing, vol. 28, no. 1, pp. 111-112, 1980.

[10] H. W. Strube, "Linear prediction on a warped frequency scale," Journal of the Acoustical Society of America, vol. 68, no. 4, pp. 1071-1076, 1980.

[11] M. Karjalainen, E. Piirilä, and A. Järvinen, "Loudspeaker response equalization using warped digital filters," in Proc. NorSig-96, pp. 367-370, Espoo, Finland, September 1996.

[12] M. Karjalainen, E. Piirilä, A. Järvinen, and J. Huopaniemi, "Comparison of loudspeaker equalization methods based on DSP techniques," Journal of the Audio Engineering Society, vol. 47, no. 1, pp. 14-31, January-February 1999.

[13] M. Karjalainen, A. Härmä, U. K. Laine, and J. Huopaniemi, "Warped filters and their audio applications," in Proc. IEEE Workshop on Applications of Signal Processing to Audio and Acoustics, New Paltz, NY, USA, October 1997.

[14] D. Preis, "Phase distortion and phase equalization in audio signal processing - a tutorial review," Journal of the Audio Engineering Society, vol. 30, no. 11, pp. 774-794, 1982.

Wee Ser received his B.Sc. (Hons) degree and Ph.D. degree, both in electrical and electronic engineering from the Loughborough University, UK in 1978 and 1982, respectively. He joined the Defence Science Organization (DSO), Singapore, as an engineer in 1982 and became the Head of the Communications Laboratory and later the Head of the Communications Research Division in 1988 and 1993, respectively. From 1995 to 1997, he was an adjunct associate professor at the School of Electrical and Electronic Engineering (EEE) in NTU (Nanyang Technological University). In 1997, he joined NTU as an associate professor and was appointed as the Director of the Centre for Signal Processing. Wee Ser was a recipient of the Colomb Plan and PSC postgraduate scholarships. He was awarded the IEE Prize during his undergraduate studies. While in DSO, he was the recipient of the prestigious Defence Technology (Individual) Prize in 1991 and an Excellence Award for a research project in 1992. $\mathrm{He}$ is a senior member of IEEE. He has published more than 40 papers in international journals and conferences, and has seven patents filed under his name. His research interests include channel equalization, space-time processing, microphone array processing, multiuser detection, noise control, and fingerprint verification techniques.

Peng Wang received his B. Eng. degree from Tsinghua University, China, in 1997, and M. Eng. degree from Nanyang Technological University, Singapore, in 2000, both in electrical engineering. He is currently a Research Engineer in Center for Signal Processing, Nanyang Technological University, Singapore. His research interests include audio processing, array processing, and advanced signal processing for communications.

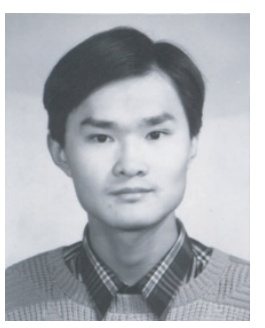


Ming Zhang received the B.Eng. and M.Eng. degrees from the National University of Defense Technology, China, in 1985 and 1988, respectively, and the Ph.D. degree from the University of Electronic Science and Technology of China in 1991, all in electrical engineering. He was a Lecturer in 1991 and then Associate Professor in 1992 with Nanjing University of Aeronautics and Astronautics, China. In September

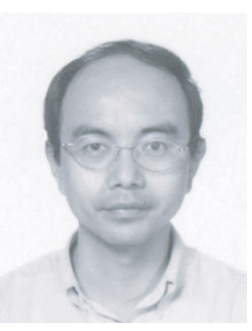
1994, he joined the School of Electrical and Electronic Engineering, Nanyang Technological University, Singapore, as a Postdoctoral Fellow. He then joined the Centre for Signal Processing as Research Fellow in June 1996 and was promoted to Senior Research Fellow in January 1998. He also received an appointment of Program Manager in June 1998 to lead a technical team on audio, speech and adaptive signal processing. In August 2001, he moved to Silicon Valley, CA, USA. After working at Sony Electronics in San Jose for one and half months, he joined Fortemedia, Inc., a start-up company on IC chip products of voice interface for mobile applications, as a Technical Director to lead the software team to develop algorithm and real-time software/firmware on DSP. He has published more than 80 papers in journals and conference proceedings and has more than ten pending patents. His research interests include adaptive signal processing, array processing, speech and audio processing and communications. Dr. Zhang received three science and technology achievement prizes from the Chinese Government and some other awards/honors. 